\title{
Microscopic Viscoelasticity: Shear Moduli of Soft Materials Determined from Thermal Fluctuations
}

\author{
F. Gittes, B. Schnurr, P.D. Olmsted*, F.C. MacKintosh, and C.F. Schmidt \\ Department of Physics 85 Biophysics Research Division, \\ University of Michigan, Ann Arbor, MI 48109-1120 \\ * Department of Physics, University of Leeds, Leeds LS2 9JT, \\ United Kingdom
}

\begin{abstract}
We describe a high-resolution, high-bandwidth technique for determining the local viscoelasticity of soft materials such as polymer gels. Loss and storage shear moduli are determined from the power spectra of thermal fluctuations of embedded micron-sized probe particles, observed with an interferometric microscope. This provides a passive, small-amplitude measurement of rheological properties over a much broader frequency range than previously accessible to microrheology. We study both F-actin biopolymer solutions and polyacrylamide (PAAm) gels, as model semiflexible and flexible systems, respectively. We observe high-frequency $\omega^{3 / 4}$ scaling of the shear modulus in F-actin solutions, in contrast to $\omega^{1 / 2}$ scaling for PAAm.

PACS numbers: 61.30.Cz, 61.30.-v, 64.70.Md, 83.70.Jr
\end{abstract}

The local analysis of viscoelasticity can explore the small-scale structure of complex fluids. It can also potentially measure bulk viscoelastic quantities in small samples. Experiments have been done to characterize the local viscoelasticity of materials since at least the 1920s, when magnetic particles in gelatin were manipulated by field gradients [1]. Crick used a similar technique to study living cells [2]. With the recent advent of methods for force generation, detection, and manipulation of particles on sub-micrometer scales, experimental possibilities have expanded greatly, and interest in microrheology has grown substantially [3 [8]. Here we report an optical technique for high resolution and high bandwidth observations of the thermal fluctuations of particles embedded in soft materials. Using dispersion relations from linear response theory, the frequency-dependent loss and elastic storage shear moduli are both calculated from the fluctuation power spectra, which we measure with a resolution of 2 Afrom $0.1 \mathrm{~Hz}$ to $20 \mathrm{kHz}$. This frequency range exceeds that of video-based microrheological experiments (although large-volume rheometers can reach frequencies up to $500 \mathrm{kHz}$ [9]) and allowed us to observe high-frequency $\omega^{3 / 4}$ scaling of the shear modulus for entangled, semiflexible solutions. These dynamics differ fundamentally from those of flexible polymer systems.

We have studied F-actin solutions as a model semiflexible polymer, and polyacrylamide (PAAm) gels as a flexible polymer control. Actin is one of the primary components of the cytoskeleton of plant and animal cells and is largely responsible for the viscoelastic response of cells [10]. Actin is a particularly accessible model system because individual filaments can be hundreds of microns in length. Viscoelastic properties of entangled F-actin solutions in vitro have been measured using conventional macroscopic rheology 11 15]. Actin has also been the subject of recent microrheological studies [3 6 .

The relationship between thermal motion and hydrodynamic response is well-known in the context of Brownian motion in simple viscous fluids. Less obviously, the bulk viscoelastic properties of complex fluids can also be determined from thermal motion. Such a passive technique is particularly useful for biopolymer systems which, on the one hand, have a limited range of linear response, but which, on the other hand, have a small enough shear modulus that the thermal motion of micron-sized probes is detectable. This idea was exploited recently by Mason and Weitz [16], who used diffusing wave spectroscopy (DWS) to observe average thermal fluctuations of concentrated suspensions of probe particles and to measure rheological quantities in complex fluids to high frequencies. That technique measures light multiply-scattered by many embedded particles, assumed to be independent. While DWS requires large samples, the present technique monitors isolated particles in very small samples.

For spherical probes, the response is given by a generalized Stokes formula, which allows for quantitative determination of macroscopic viscoelastic moduli. In our experiments, rigid spherical beads of radius $R$ move in a viscoelastic medium consisting of polymer plus solvent. When a force $f$ acts on such a sphere, the deformation of the surrounding medium can be calculated [17] to obtain the linear response for the sphere: $x_{\omega}=\alpha(\omega) f_{\omega}$, where $x$ is the position of the sphere. In general, the response function is complex: $\alpha(\omega)=\alpha^{\prime}(\omega)+i \alpha^{\prime \prime}(\omega)$. Within certain limits described below, this response function reflects pure shear motion of the medium and is given by

$$
\alpha(\omega)=\frac{1}{6 \pi G(\omega) R} .
$$

Here, the frequency-dependent complex shear modulus is 
given by $G(\omega)=G^{\prime}(\omega)+i G^{\prime \prime}(\omega)$, where $G^{\prime}$ and $G^{\prime \prime}$ are the storage and loss modulus. (For the case of a purely viscous fluid $G=-i \omega \eta$, leading to the well-known Stokes formula $f=6 \pi \eta R \dot{x})$. The fluctuation-dissipation theorem [18] relates $\alpha$, and thus $G$, to Brownian motions of the bead induced by thermal fluctuations of the medium.

As in the case of a viscous fluid [19], Eq. (11) is valid provided that the viscous penetration $\operatorname{depth} \delta=\sqrt{2 \eta / \rho \omega}$ is large compared with $R$, where $\rho$ and $\eta$ are solvent density and viscosity; this is true for frequencies up to about $1 \mathrm{MHz}$ for a $1 \mu \mathrm{m}$ bead in water. The validity of continuum viscoelasticity also requires that the bead is at least larger than the mesh size of the network.

A somewhat more subtle limitation on all microrheological methods to date, including ours, concerns the two-component nature of polymer solutions. In a general viscoelastic medium, there can be another relevant material parameter in addition to the shear modulus: the compression modulus, or equivalently, the Poisson ratio. In a simple viscous fluid, only shear stress is relevant because of the incompressibility of the fluid. In a polymer solution consisting of both network and solvent, this is also true for the high-frequency response because the network and incompressible solvent become strongly coupled. While a rigorous calculation of the response requires a full treatment including both network and solvent aspects of the material, we can estimate the validity of the approach given above within a so-called two fluid model [20], which takes into account the viscous coupling of the two components. We estimate the frequency crossover from the balance between viscous and elastic forces. The viscous force of the solvent on the network is $\eta v / \xi^{2}$ per volume, where $\xi$ is the mesh size and $v$ is the solvent velocity relative to the mesh. The local elastic force in the network is $G \nabla^{2} u \sim G u / R^{2}$ per volume at the bead surface, where $u$ is the network displacement field. Thus viscous coupling is dominant, and solvent and network move together, above a frequency

$$
\omega_{c} \simeq G \xi^{2} / \eta R^{2} .
$$

Above $\omega_{c}$, Eq. (11) will hold. In our experiments $\omega_{c}$ is of order $10 \mathrm{~Hz}(G \simeq 1 \mathrm{~Pa}, \xi / R \simeq 0.1, \eta=1 \mathrm{cP})$, which is at the lower limit of our instrumental resolution. At frequencies $\omega \ll \omega_{c}$, the response includes compressional modes not present in Eq. (1); these will have wavelengths $q^{-1} \sim \xi(G / \eta \omega)^{1 / 2}$ and will make $\alpha(\omega)$ independent of bead size.

Applying the fluctuation-dissipation theorem to the power spectral density (PSD) of bead displacements $\left\langle x_{\omega}^{2}\right\rangle$ yields

$$
\alpha^{\prime \prime}(\omega)=\frac{\omega}{2 k T}\left\langle x_{\omega}^{2}\right\rangle
$$

When $\alpha^{\prime \prime}(\omega)$ is obtained over a large enough range of frequencies, the complex response $\alpha(\omega)$ is obtained from the Kramers-Kronig relations [18] by evaluating the dispersion integral

$$
\alpha^{\prime}(\omega)=\frac{2}{\pi} P \int_{0}^{\infty} d \zeta \frac{\zeta \alpha^{\prime \prime}(\zeta)}{\zeta^{2}-\omega^{2}}
$$

The principal-value integral $P$ in Eq. (1) is conveniently computed on a discrete data set as a sine transform of $\alpha^{\prime \prime}(\omega)$ followed by a cosine transform. We finally take the complex reciprocal of $\alpha(\omega)$ to obtain the complex $G(\omega)$ by Eq. (1). We have evaluated Eq. (4) using model spectra with various upper and lower cutoffs [17 and found it to be reliable over the scaling range discussed below.

The shear modulus $G(\omega)$ typically exhibits three distinct regimes in entangled flexible polymer solutions [21]. For a non-crosslinked solution the behavior is essentially that of a viscous liquid at frequencies lower than $1 / \tau_{R}$, where $\tau_{R}$ is the reptation time. Above $1 / \tau_{R}$ a rubber-like plateau appears, with a dominant, frequencyindependent elastic response as in a crosslinked gel. At even higher frequencies, above the relaxation rate of the mesh [21], the high-frequency moduli $G^{\prime}$ and $G^{\prime \prime}$ are expected to obey a pure power-law, since no new characteristic time appears until the molecular cutoff is reached.

Our samples were prepared as follows. Actin was extracted from chicken skeletal muscle according to standard procedures [22], combined with silica beads, and polymerized (buffer: 2 mM HEPES ( $\mathrm{pH}$ 7.0), $2 \mathrm{mM}$ $\mathrm{MgCl}_{2}, 50 \mathrm{mM} \mathrm{KCl}, 1 \mathrm{mM}$ EGTA, $1 \mathrm{mM}$ ATP) at a concentration of $2 \mathrm{mg} / \mathrm{ml}$ under slow rotation to avoid bead sedimentation. This yields a highly entangled solution of stiff filaments with persistence lengths of order $10 \mu \mathrm{m}$ and a mesh size $\xi \cong 0.2 \mu \mathrm{m}$ [23]. PAAm gels were prepared as in standard gel electrophoresis [24] with concentrations of 2, 2.5, and $3 \%$ weight/volume (20,25, and $30 \mathrm{mg} / \mathrm{ml}$ ); the PAAm contained $3 \%$ bis-acrylamide as a crosslinker. These flexible polymers have a persistence length on the order of nanometers; the mesh size of a $2 \%$ gel is about $50 \AA$ 25]. The smallest sample dimension was their thickness of $70 \mu \mathrm{m}$ (F-actin) and $140 \mu \mathrm{m}$ (PAAm).

For bead position detection we couple a near-infrared laser into an optical bench-mounted differential interference contrast (DIC) microscope. Polarized at $45^{\circ}$ to the axis of the DIC Wollaston prism, the beam is split and the microscope objective produces two overlapping foci $(\sim 200 \mathrm{~nm}$ apart) of orthogonal polarizations [26]. A bead of higher refractive index than the medium in the double focus causes a differential phase shift $\Delta \phi(x)$ between the beams which is a measure of bead position $x$. After recombination by a second Wollaston prism, the light, now elliptically polarized, is passed through a quarter-wave plate and a polarizing beam splitter onto two photodiodes. The normalized difference of the photodiode currents is $\sin (\Delta \phi)$. Small excursions of a centered 
bead result in a proportional voltage response which we calibrated by controlled displacements of a piezoelectric stage. This set-up is also used for optical trapping; here, the laser focus was broadened and the power reduced ( $\sim 0.6 \mathrm{~mW}$ in the specimen) so that optical forces were negligible. Our resolution at high frequencies was limited by shot noise to about $2 \times 10^{-6} \mathrm{~nm}^{2} \mathrm{~Hz}^{-1}$. The beadposition signal was digitized at $60 \mathrm{kHz}$ and filtered above $20 \mathrm{kHz}$ to eliminate aliasing.

In actin solutions, the power spectral density (PSD) of bead fluctuations $\left\langle x_{\omega}^{2}\right\rangle$ was highly reproducible in shape and amplitude, not only for different beads within a sample but between samples and between different actin preparations [17. For samples with beads of diameter $0.9,2.1$, and $5.0 \mu \mathrm{m}$, the variation in PSD among six beads per sample (at $100 \mathrm{~Hz}$ ) was $10-20 \%$.

The fluctuation power spectra of beads in actin are shown in Fig. 11. Above about $20 \mathrm{~Hz}$, the PSDs scale (roughly) with bead size, as predicted by Eq. (11). All spectra show a slight downturn above $3 \mathrm{kHz}$, which is currently an experimental limitation. At low frequencies, the slopes decrease in a bead-size dependent way. We believe this to be the transition from a regime of pure shear fluctuations to long-wavelength compressional modes, as predicted by Eq. (2). For $5.0 \mu \mathrm{m}$ beads, the PSD displays power-law behavior $\left(\sim \omega^{-n}\right.$ with $\left.n=1.77 \pm 0.02\right)$ over almost three decades of frequency. In contrast, $0.8 \mu \mathrm{m}$ polystyrene beads in glycerol, a simple viscous fluid, should display a diffusional exponent of $n=-2$; we find $n=1.94 \pm 0.03$. Furthermore, from this spectrum we obtain a value of $11.3 \mathrm{P}$, compared to a tabulated value of $9.3 \mathrm{P}$ at $25^{\circ} \mathrm{C}$.

The $G^{\prime}(\omega)$ and $G^{\prime \prime}(\omega)$ calculated from the spectra for $5.0 \mu \mathrm{m}$ beads are shown in Fig. 11B. These beads are about 20 times larger than the mesh size, so that the continuum model of Eq. (1) applies: as expected, a powerlaw has emerged in $G^{\prime}$ and $G^{\prime \prime}$. No low-frequency plateau is observed. (In 14 this plateau was found below about $0.01 \mathrm{~Hz}$ at similar concentrations.) The scaling corresponds, via Eqs. (11) and (4), to a complex power-law $G(\omega) \propto(i \omega)^{z}$, so that $G^{\prime \prime} / G^{\prime}=\tan \pi z / 2$. This ratio allows an estimate of $z=n-1=0.76 \pm 0.02$ between $10 \mathrm{~Hz}$ and $100 \mathrm{~Hz}$, consistent with the power observed in the PSD.

Other studies of actin rheology [4,14, at frequencies below about $3 \mathrm{~Hz}$, have reported $G \sim \omega^{1 / 2}$. However, at these lower frequencies, their results may reflect an incomplete transition to the $\omega^{3 / 4}$ scaling regime. The magnitudes we measure for $G^{\prime}$ and $G^{\prime \prime}$ are consistent with other experiments [3, 4,15 , although discrepancies persist in the literature 12$]$. Amblard et al. [6] found bead-diffusion dynamics in more dilute actin solutions consistent with our scaling results, but suggest an explanation based on isolated filament dynamics. We believe that at our higher concentrations, for which the mesh size is substantially smaller than the bead diameter, a continuum elastic approach as described here is correct.
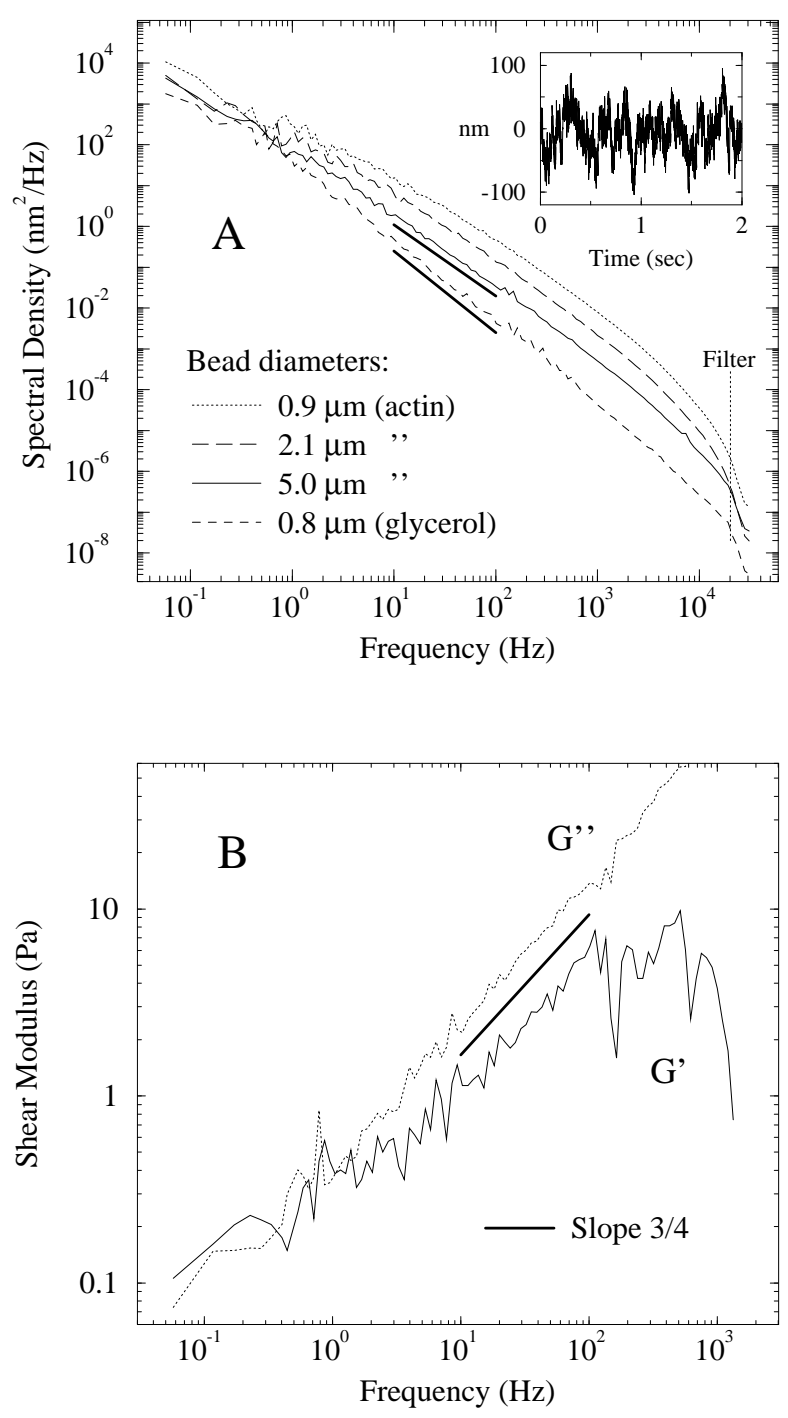

FIG. 1. (A): Power spectral density (PSD) of thermal bead motion in an actin solution $(2 \mathrm{mg} / \mathrm{ml})$, averaged over several beads and within bins of equal log width. The PSD of a bead in glycerol is shown for comparison. The indicated slopes are -1.75 and -2.0 . Inset: Time series data for a $0.9 \mu \mathrm{m}$ bead. For the smaller beads, deviations from scaling may indicate a failure of the continuum assumption. (B): Real part (solid) and imaginary part (dotted) of the complex modulus $G=G^{\prime}(\omega)+i G^{\prime \prime}(\omega)$ calculated from the spectrum in (A) (5.0 $\mu \mathrm{m}$ bead). $G^{\prime}$ and $G^{\prime \prime}$ scale as $\omega^{z}$, with $z=0.76 \pm 0.02$ determined from their ratio. The downturn in the spectra at about $20 \mathrm{kHz}$ is due to an anti-aliasing filter.

The fluctuation spectra of $0.9 \mu \mathrm{m}$ silica beads in 2 , 2.5, and $3 \%$ crosslinked PAAm gels are shown in Fig. 22. The mesh size of this gel is about $50 \AA$ 25] so that a continuum model easily applies. The $2 \%$ spectrum has a slope of about -1.5 near $100 \mathrm{~Hz}$, consistent with the Rouse model and in clear contrast to the actin solutions. 
The $2 \%$ spectrum decreases in slope below about $2 \mathrm{~Hz}$ due to the onset of an elastic plateau in $G^{\prime}$ (the other spectra reflect a plateau over most of their range, aside from the low-frequency instrumental noise from drift below a few $\mathrm{Hz}$ ). The calculated $G^{\prime}(\omega)$ are shown explicitly in Fig. 2 lower inset, where the plateau values of $G^{\prime}$ are $2.0 \mathrm{~Pa}(2 \%), 24 \mathrm{~Pa}(2.5 \%)$ and $100 \mathrm{~Pa}(3 \%)$. Because the plateau extends to such high frequencies, $G^{\prime}(\omega)$ does not yet exhibit the $G(\omega) \sim \omega^{1 / 2}$ scaling [21] as suggested by the PSD slope of -1.5 . Close to the gelation threshold, the shear modulus increases very rapidly with concentration, as also found by others 27 .

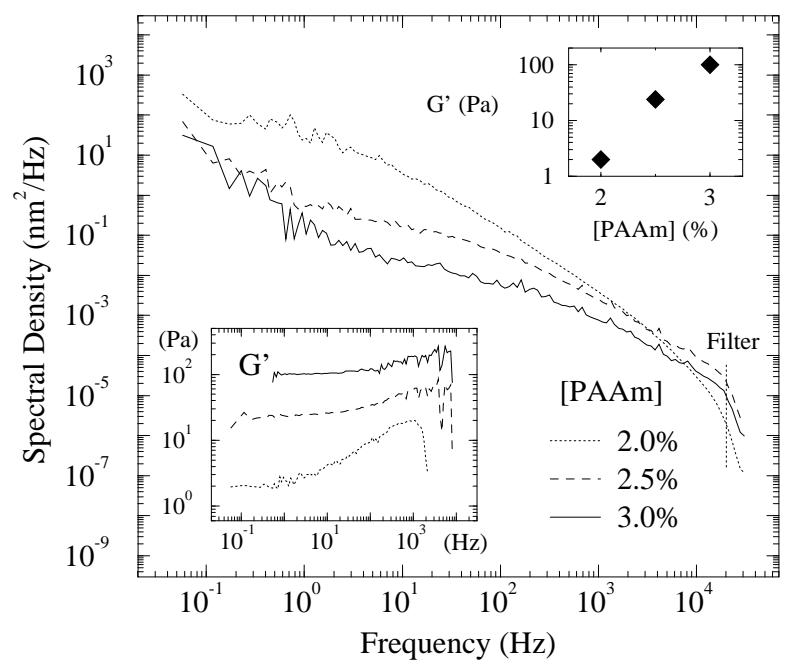

FIG. 2. Power spectra of thermal motion of $0.9 \mu \mathrm{m}$ beads in polyacrylamide gels of different concentrations, averaged as in Fig. 1A, cut off by an anti-aliasing filter at $20 \mathrm{kHz}$. Lower inset: The elastic modulus $G^{\prime}(\omega)$ obtained from each of these spectra, as in Fig. 11B. Upper inset: Plateau moduli (values at $1 \mathrm{~Hz}$ ), as a function of concentration, of the three curves in the lower inset.

The present technique differs in its spatial resolution and wide frequency range from prior microrheological studies such as those of Refs. [3 [6] employing video microscopy. The extended frequency range has allowed us to determine the high-frequency scaling of the shear modulus for actin well above the elastic plateau. We have described the inherent limitations in this and similar microrheology studies at low frequencies. For rather sparse networks, the low-frequency response cannot be described by a shear modulus alone, but must also take into account the local compression of the network; this may invalidate the generalized Stokes formula, Eq. (1), for frequencies accessible to video. In comparison to most commercial rheometers, our technique allows the study of soft materials and small sample volumes, which may be particularly useful in biological applications.

This work was supported in part by the Whitaker
Foundation, the National Science Foundation (Grant Nos. BIR 95-12699 and DMR 92-57544), and by the donors of the Petroleum Research Fund, administered by the ACS. We acknowledge generous technical support from the Rowland Institute for Science, particularly W. Hill. We thank P. Janmey, J. Käs, and D. Weitz for helpful discussions. PDO and FCM acknowledge support from NATO Grant No. CRG 960678. FCM wishes to thank the Aspen Center for Physics.

[1] H. Freundlich and W. Seifriz, Z. Phys. Chem. 104, 233 (1922).

[2] F. Crick and A. Hughes, Exp. Cell Res. 1, 37 (1950).

[3] K.S. Zaner and P.A. Valberg, J. Cell Biol. 109, 2233 (1989); also reviewed in F.C. MacKintosh and P.A. Janmey, Curr. Opin. Sol. State. Mat. Sci., in press (1997).

[4] F. Ziemann, J. Rädler, and E. Sackmann, Biophys. J. 66, 2210 (1994).

[5] F.G. Schmidt, F. Ziemann, and E. Sackmann, Eur. Biophys. J. 24, 348 (1996).

[6] F. Amblard et al., Phys. Rev. Lett. 77, 4470 (1996).

[7] B. Schnurr, F. Gittes, P.D. Olmsted, C.F. Schmidt, F. C. MacKintosh, In Mat. Res. Soc. Symp. Proc. R.H. Austin, T.C. Halsey, and D. Wirtz (Ed.), 1997.

[8] T.G. Mason, A. Dhople, and D. Wirtz In Mat. Res. Soc. Symp. Proc. R.H. Austin, T.C. Halsey, and D. Wirtz (Ed.), 1997; T.G. Mason, K. Ganesan, D. Wirtz, S.C. Kuo, preprint.

[9] T.M. Stokich et al., J. Rheol. 38, 1195 (1994).

[10] T.P. Stossel, Sci. Am. 271, 54 (1994).

[11] T.D. Pollard, I. Goldberg, W.H. Schwarz, J. Biol. Chem. 267, 20339 (1992).

[12] P.A. Janmey et al., J. Biol. Chem. 269, 32503 (1994).

[13] J. Käs et al., Biophysical Journal 70, 609 (1996).

[14] O. Müller et al., Macromolecules 24, 3111 (1991); R. Ruddies et al., Eur. Biophys. J. 22, 309 (1993).

[15] Reviewed in P.A. Janmey, Curr. Opin. Cell Biol. 2, 4 (1991).

[16] T.G. Mason and D.A. Weitz, Phys. Rev. Lett. 74, 1250 (1995).

[17] F. Gittes et al. to appear; B. Schnurr et al. to appear.

[18] L.D. Landau, E.M. Lifshitz, and L.P. Pitaevskii, Statistical Physics (Pergamon Press, New York, 1980).

[19] L.D. Landau and E.M. Lifshitz, Fluid Mechanics (Pergamon Press, Reading, 1959).

[20] F. Brochard and P.G. de Gennes, Macromolecules 10, 1157 (1977); S.T. Milner, Phys. Rev. E 48, 3674 (1993).

[21] M. Doi and S.F. Edwards, The Theory of Polymer Dynamics (Clarendon Press, Oxford, 1988).

[22] J.D. Pardee and J.A. Spudich, Meth. Enzymol.85, 164 (1982).

[23] C.F. Schmidt et al., Macromolecules 22, 3638 (1989).

[24] Bio-Rad Laboratories, US/EG Bulletin 1156.

[25] J.S. Fawcett and C.J.O.R. Morris, Separation Sci.1, 9 (1966).

[26] W. Denk and W.W. Webb, Appl. Opt. 29, 2382 (1990); K. Svoboda et al., Nature 365, 721 (1993).

[27] R. Nossal, Rubber Chem. Technol. 61, 255 (1988). 